\title{
Gestantes em uso de substâncias psicoativas atendidas por enfermeiros na Atenção Primária à Saúde
}

\author{
Angela Aparecida Peters ${ }^{1}$ \\ (D) https://orcid.org/0000-0002-0363-8371 \\ Hugo Ramalho Cruzeiro² \\ (D) https://orcid.org/0000-0002-5229-4034 \\ Otavia Gonçalves Paulino Bertolini² \\ (D) https://orcid.org/0000-0002-0784-7939 \\ Giselle de Paula Assis ${ }^{3}$ \\ (D) https://orcid.org/0000-0002-5105-4960 \\ Adriana Dias Silva ${ }^{4}$ \\ (D) https://orcid.org/0000-0002-1353-6723 \\ Maria Angélica de Almeida Peres 5 \\ (D) https://orcid.org/0000-0002-6430-3540
}

\footnotetext{
${ }^{1}$ Escola de Enfermagem Anna Nery, NUPHEBRAS, Rio de Janeiro, RJ, Brasil.

2 Faculdade de Ciências Médicas, Suprema, Juiz de Fora, MG, Brasil.

${ }^{3}$ Universidade Federal de Juiz de Fora, Juiz de Fora, MG, Brasil.

${ }^{4}$ Fundação Universidade Federal de Rondônia, Departamento de Enfermagem, Porto Velho, RO, Brasil.

${ }^{5}$ Universidade Federal do Rio de Janeiro, Escola de Enfermagem Anna Nery, Rio de Janeiro, RJ, Brasil.
}

Objetivo: discorrer sobre o pré-natal realizado por enfermeiros na Atenção Primária à Saúde a gestantes usuárias de substâncias psicoativas. Método: estudo descritivo-exploratório, de abordagem qualitativa, realizado em seis Unidades de Atenção Primária à Saúde em um município de Minas Gerais. A coleta de dados ocorreu por meio de entrevista semiestruturada com nove enfermeiros que realizavam consultas de pré-natal. A técnica de análise do conteúdo foi utilizada para o tratamento dos dados. Resultados: dificuldades com a adesão das gestantes ao pré-natal e sua participação em grupos educativos. Os enfermeiros também identificaram as substâncias psicoativas mais utilizadas pelas gestantes e relataram abordar os malefícios do uso das substâncias, a fim de estimularem a decisão pela redução. Conclusão: o interesse das gestantes pelo tratamento foi pequeno e resultou na baixa adesão aos programas oferecidos na Atenção Primária, o que se revelou um problema de saúde pública, que requer práticas reorientadas de atenção a esta clientela.

Descritores: Gestantes; Consumo de Bebidas Alcoólicas; Drogas Ilícitas; Assistência de Enfermagem; Atenção Primária à Saúde.

\section{Como citar este artigo}

Peters AA, Cruzeiro HR, Bertolini OGP, Assis GP, Silva AD, Peres MAA. Pregnant women using psychoactive substances attended by nurses in Primary Health Care. SMAD, Rev Eletrônica Saúde Mental Álcool Drog. 2020;16(2):1-9. doi: https://dx.doi.org/10.11606/issn.1806-6976.smad.2020.166357 


\title{
Pregnant women using psychoactive substances attended by nurses in Primary Health Care
}

\begin{abstract}
Objective: to discuss prenatal care performed by nurses in Primary Health Care for pregnant women who use psychoactive substances. Method: a descriptive-exploratory study, with a qualitative approach, carried out in six Primary Health Care Units in a city in Minas Gerais. Data collection took place through semi-structured interviews with nine nurses who performed prenatal consultations. The content analysis technique was used for data treatment. Results: difficulties with pregnant women's adherence to prenatal care and their participation in educational groups. Nurses also identified the psychoactive substances most used by pregnant women and reported addressing the harms of substance use, to encourage the choice to reduce use. Conclusion: the pregnant women's concern about treatment was poor and resulted in low adherence to the programs offered in Primary Care, which proved to be a public health problem, which requires reoriented care practices for this clientele.
\end{abstract}

Descriptors: Pregnant Women; Alcohol Drinking; Street Drugs; Nursing Care; Primary Health Care.

\section{Embarazadas que usan sustancias psicoactivas atendidas por enfermeros en la Atención Primaria a la Salud}

Objetivo: discurrir sobre o prenatal realizado por enfermeros en la Atención Primaria a la Salud a embarazadas usuarias de substancias psicoactivas. Método: estudio descriptivo exploratorio, de abordaje cualitativo, realizado en seis Unidades de Atención Primaria a la Salud en un municipio de Minas Gerais. La recogida de datos ocurrió por medio de entrevista semiestructurada con nueve enfermeros que realizaban consultas de prenatal. La técnica de análisis del contenido fue utilizada para el tratamiento de los datos. Resultados: dificultades con la adhesión de las embarazadas al prenatal y su participación en grupos educativos. Los enfermeros también identificaron las sustancias psicoactivas más utilizadas por las embarazadas y relataron abordar los daños del uso de las sustancias, a fin de estimular la decisión por la reducción. Conclusión: el interés de las embarazadas por el tratamiento fue pequeño y resultó en baja adhesión a los programas ofrecidos en la Atención Primaria, lo que se reveló un problema de salud pública, el que requiere prácticas reorientadas de atención a esta clientela.

Descriptores: Mujeres Embarazadas; Consumo de Bebidas Alcohólicas; Drogas Ilícitas; Atención de Enfermería; Atención Primaria de Salud. 


\section{Introdução}

A relação gestação e substância psicoativa (SPA) é um problema de saúde pública mundial. Em alguns países como Nova Zelândia e Inglaterra, a prevalência do uso de álcool é $56 \%$ e $75 \%$, respectivamente ${ }^{(1)}$, enquanto no Brasil foi de $34,4 \%{ }^{(2)}$ e esse uso teve associação com diabetes gestacional, ideação suicida e consumo de tabaco(3). Em um estudo de revisão, também foi encontrada prevalência de outras SPAs como crack-cocaína em diversas partes do mundo(4).

O uso de SPA e gestação é complexo, requer atendimento direcionado à sua redução e suspensão. A atuação da Atenção Primária à Saúde (APS) é fundamental para um melhor desenvolvimento do ciclo gravídico-puerperal(5-6).

Durante a consulta de pré-natal na APS, deve ser realizada uma investigação criteriosa dos antecedentes familiares e hábitos da gestante. Em caso de histórico para SPA, deve haver uma avaliação detalhada com vistas à detecção de uso crônico ou situações de risco, como intoxicações agudas, risco de suicídio, autoagressão e comorbidades psiquiátricas(5). Além disso, os malefícios acarretados pelo uso de SPA no desenvolvimento do feto devem ser apontados, para conscientizar a gestante sobre sua corresponsabilidade no sucesso da gestação(5-7).

O trabalho com gestantes usuárias de SPA se dá através de acompanhamentos periódicos e sua inserção em grupos de apoio específicos, incluindo os familiares. Tal direcionamento é extremamente importante para captar a gestante e sua família para o pré-natal, e o enfermeiro é um dos profissionais responsáveis por interferir direta ou indiretamente para um cuidado adequado na $\operatorname{APS}^{(5)}$.

O cuidado com gestantes dependentes de SPA é desafiador e exige preparo especial por parte dos enfermeiros da APS para orientar e incentivar a abstinência completa e duradoura de todas as substâncias, e oferecer suporte para que essa transição ocorra de forma segura e tolerável pelas gestantes, dada a dificuldade de manter a abstinência em casos de dependência química(6,8). Além dos exames clínicos, o enfermeiro deve reservar um tempo para a escuta subjetiva e oferta de orientações, criando um vínculo maior com as gestantes e seus familiares, com o objetivo de construir uma relação de confiança(5).

O consumo de SPA durante a gestação remete a uma preocupação constante das equipes da APS, no que tange sua assistência e prevenção. Uma vez detectado durante as consultas de pré-natal, pode ser que o problema não se resolva durante a gravidez, restando ao médico ou enfermeiro encaminhar a usuária para acompanhamento em serviços especializados, o que não descaracteriza a APS do seu papel de coordenação do cuidado(5) $^{(5)}$.
Porém, o encaminhamento pode causar um rompimento no vínculo entre a APS e a gestante, que já tem um perfil frágil por si só. Sem tal vínculo, pouca coisa será possível, caso a APS, como representação do território geográfico e afetivo, perca a sua corresponsabilização neste acompanhamento, à medida que deixe de lançar mão de ações singulares para garantir a integralidade em ações interinstitucionais, como as de redução de danos e educação em saúde e as que objetivam a otimização dos profissionais de saúde, tanto da baixa quanto da média complexidade, na prevenção dos agravos da gestação(9).

Este estudo tem como objetivo discorrer sobre o atendimento pré-natal para gestantes usuárias de substâncias psicoativas, realizado por enfermeiros em Unidades de Atenção Primária à Saúde-UAPS. Espera-se contribuir para a construção de bases teóricas para reorientar as práticas desenvolvidas nas UAPS no atendimento às gestantes e para o desenvolvimento de estratégias de intervenção, acompanhamento e educação em saúde, evitando riscos e agravamentos e possibilitando uma melhor qualidade de vida para a população assistida.

\section{Método}

Trata-se de um estudo descritivo-exploratório de abordagem qualitativa. As metodologias de pesquisa qualitativas incorporam a questão do significado e da intencionalidade como inerentes aos atos, relações humanas e estruturas sociais como construções humanas significativas(10-11).

A etapa de campo foi conduzida em fevereiro e março de 2016, em seis Unidades de Atenção Primária de Saúde (UAPS) parte do Projeto Integrador (PI) de ensino-pesquisa entre a Faculdade de Ciências Médicas e da Saúde - Suprema e a Secretaria Municipal de Saúde da Prefeitura Municipal de Juiz de Fora. Estas integram um dos componentes centrais de ensino-aprendizagem, alocado na estrutura curricular dos cursos da área da saúde na presente instituição. O PI tem papel de relevo na tríade ensino-pesquisa e extensão, pois incorpora a comunidade acadêmica ao meio social local e regional, com objetivo de contribuir para a formação integrada dos profissionais de saúde através da inserção de estudantes em diferentes comunidades. As seis UAPS tinham aproximadamente três equipes da Estratégia Saúde da Família. Como os estudantes do $8^{\circ}$ período de Enfermagem estavam sob estágio supervisionado nas respectivas UAPS, que eram os cenários de prática do PI, os enfermeiros selecionados para o estudo foram os supervisores credenciados, que acompanhavam esses estudantes no campo de prática na realização do pré-natal.

A coleta de dados foi realizada por meio de roteiro para entrevista semiestruturada e um formulário com questões para caracterização dos participantes, incluindo 
gênero, tempo de formação e especialização. Questões norteadoras compuseram o roteiro com perguntas a respeito de como ocorria o primeiro atendimento de prénatal às gestantes em uso de SPA nas UAPS; tipos de drogas mais utilizados por estas; estratégias e condutas adotadas pelos enfermeiros; e práticas educativas desenvolvidas em relação ao uso de SPA.

Os critérios de inclusão compreenderam enfermeiros das UAPS que realizavam a consulta de prénatal e supervisionavam alunos do PI. Os critérios de exclusão foram todos os enfermeiros de férias ou licença médica no período da coleta de dados. As entrevistas foram realizadas pelos próprios pesquisadores com nove enfermeiros nas UAPS, em sala reservada para este fim, fora do horário de trabalho e após agendamento prévio por telefone. Os participantes foram esclarecidos sobre o tema e os objetivos da pesquisa, e solicitada sua autorização para gravar a entrevista. A duração média de cada entrevista foi de aproximadamente 30 minutos. $\mathrm{O}$ anonimato dos participantes foi garantido mediante a utilização de códigos alfanuméricos (E1, E2...).

A análise dos dados segundo as orientações de Laurence Bardin(12) foi composta por três grandes etapas: pré-análise; exploração do material e tratamento de resultados. A Pré-análise foi fase de organização, onde ocorreu a leitura flutuante e elaboração de indicadores que fundamentaram a interpretação. Na Exploração do material, as unidades de registro identificadas na etapa anterior foram organizadas visando a categorização. O Tratamento dos resultados compreendeu a inferência e interpretação.

Após a coleta de dados, as entrevistas gravadas em mídia MP3 foram transcritas na íntegra. Para a organização dos dados, foi utilizado o software Microsoft Excel. A partir dos objetivos do estudo e da leitura flutuante realizada, foram elaboradas perguntas analíticas. Para a análise e interpretação do conteúdo, os discursos foram explorados e utilizados como respostas às questões essenciais e problematizadoras constantes das entrevistas. A partir disso, foram destacadas as unidades de registro, que possibilitaram a criação de categorias.

Seguindo todos os procedimentos legais, este estudo foi apreciado e aprovado pelo Comitê de Ética em Pesquisa da Faculdade de Ciências Médica e da Saúde-Suprema sob o Parecer Consubstanciado de no 1.373.578 e CAAE 51602815.0.0000.5103.

\section{Resultados}

Dos nove participantes do estudo, dois eram do sexo masculino e sete do sexo feminino, com idade entre 31 a 54 anos e média de idade de 44,2. O tempo de formação desses participantes foi de dois a 28 anos. Todos profissionais eram especialistas em alguma área: um em Enfermagem Obstétrica; quatro em Enfermagem em Saúde da Família; três em Enfermagem em Centro de Tratamento Intensivo e Neonatal; e um em Enfermagem do Trabalho.

Por meio dos discursos dos participantes, foi possível construir três categorias temáticas: A) Atendimento de pré-natal nas UAPS com gestantes usuárias de álcool, crack e outras drogas; B) Drogas mais utilizadas pelas gestantes e os riscos associados; e C) Condutas adotadas pelos enfermeiros com as gestantes usuárias de drogas e álcool nas UAPS.

A) Atendimento de pré-natal nas Unidades de Atenção Primária de Saúde com gestantes usuárias de álcool, crack e outras drogas:

Durante o pré-natal, ao serem identificados um ou mais fatores de risco, as gestantes eram encaminhadas para ambulatórios de alto risco para as devidas avaliações de seguimento e acompanhamento do pré-natal, seguindo um fluxo de atendimento multiprofissional em consultas intercaladas no ambulatório de risco e na UAPS: ... Encaminhamos também para assistente social, que faz esse encaminhamento para o CAPS. Depois da primeira consulta, ela (a gestante) é encaminhada para o pré-natal de risco; depois disso, ela retorna fazendo consultas intercaladas com enfermeiro, médico e atenção secundária ... (E4); ...Elas são referenciadas para o alto-risco; o próprio serviço da atenção secundária solicita o acompanhamento da atenção primária, ou seja, ela passa uma consulta com o médico de lá e intercala com um médico daqui ... (E8); ... As gestantes usuárias de drogas, nós as encaminhamos para o alto-risco; mas nós tanto acompanhamos no alto-risco, como aqui; não só com as usuárias de drogas, mas como qualquer gestante de alto-risco, nós continuamos acompanhando aqui; É uma consulta no alto-risco e uma consulta na Unidade... (E3).

B) Drogas mais utilizadas pelas gestantes e os riscos associados

Para o atendimento de pré-natal às gestantes usuárias de substâncias psicoativas, o enfermeiro precisava ter conhecimento das especificidades de cada substância e assim, traçar um plano de cuidado com ação preventiva, de modo que os riscos fossem reduzidos e a gestante tivesse mais chances de uma gravidez segura. As drogas utilizadas pelas gestantes atendidas nas UAPS pesquisadas foram: maconha, tabaco, crack e cocaína.

De acordo com a área de atendimento de cada UAPS, os enfermeiros destacaram as drogas mais utilizadas, conforme apontado nas frases: Geralmente, eu atendia usuárias de maconha, crack e álcool; hoje em dia, o abuso é da maconha e crack ... (E2); Álcool, cigarro (de nicotina) e maconha; há uma parte que utiliza cocaína também, porém, ultimamente há o crack, que é uma droga devastadora, muito barata e de fácil acesso; então, infelizmente, o crack hoje tem dominado ... (E5); ... Oficialmente, eu soube que se usava o crack, maconha e tabaco; tabaco é muito comum; agora o álcool é mais difícil ... (E7); ... Os tipos de drogas mais utilizados por elas aqui, ou é 
o cigarro (de nicotina) ou o crack; o álcool nem tanto porque banalizam; elas não consideram nem tanto como droga; então, tabaco, maconha e crack são os mais utilizados ... (E8).

C) Condutas adotadas pelos enfermeiros com as gestantes usuárias de drogas e álcool nas UAPS

Os resultados apresentados nesta categoria procuraram traduzir as condutas adotadas e as dificuldades encontradas pelos enfermeiros para desenvolverem práticas educativas com as gestantes usuárias de substâncias psicoativas, conforme observado:

Aqui na UAPS, nós temos grupos de tabagismo, e quando nós vemos que não há adesão, que as gestantes não estão comparecendo, nós as encaminhamos para o CAPS, onde os profissionais darão o direcionamento ... (E1); ... Temos grupo de tabagismo; tem CAPS álcool e drogas, que nós encaminhamos também, e damos orientações durante o pré-natal, em que falamos com as gestantes durante todo tempo; apesar de umas serem bem difíceis ... (E3); A maioria dessas gestantes (usuárias de álcool e outras drogas) não frequentam direito o pré-natal; então acaba que nós temos que fazer uma busca ativa e tentar sensibilizar a família para trazer ... (E7); Aqui na unidade, nós ainda não fazemos esse tipo de trabalho com essas gestantes; trabalhamos com elas esses malefícios normalmente durante as consultas; a gente tem uma certa dificuldade até com as gestantes normais em manter aqui na unidade esse tipo de trabalho educativo, pois elas não são assíduas ... (E8).

$\mathrm{O}$ estudo mostrou que o cuidado realizado pelos enfermeiros nas UAPS ocorreu no atendimento pré-natal, e houve dificuldades com a manutenção da frequência das gestantes nas consultas e nos grupos educativos.

Os dados mostraram que as ações educativas realizadas por enfermeiros ocorreram mais no âmbito individual, direcionadas às gestantes usuárias de algum tipo de SPA. Na visão dos enfermeiros, as gestantes apresentavam muita resistência e dificuldades para aderir aos grupos educativos: ... Normalmente, as gestantes não relatam o uso de álcool e drogas na primeira consulta; relatam no terceiro e quarto mês com muita insistência do profissional; há uma dificuldade muito grande para elas assumirem o uso da droga ou de qualquer outra substância... (E2); Nós aproveitamos a vinda delas para a consulta e fazemos todo o educativo; elas não vêm participar de grupo de gestantes; elas não têm essa paciência e interesse em fazer; então, nós fazemos toda educação no pré-natal: as necessidades, os riscos e os cuidados que elas devem ter durante as consultas de pré-natal... (E4).

Ainda nessa perspectiva, foram percebidas as dificuldades dos enfermeiros em manter contato direto e contínuo com as gestantes, pois quando identificavam o uso de SPA, encaminhavam a gestante para o alto risco, e algumas delas não retornavam mais para a APS, dificultando o vínculo terapêutico que proporcionava as práticas educativas. Os relatos a seguir ilustram esta questão: Esse vínculo (enfermeiro-gestante) é difícil, porque nós até tentamos, mas na maioria dos casos, elas abandonam o pré-natal; mas quando fazem aqui, nós mantemos sim ... (E7); ... infelizmente, a realidade é que quando vai para o alto risco, ela não tem mais nada a ver comigo... (E8).

Nestes casos, foi identificada a dificuldade para a continuidade da assistência a essas gestantes; da parte delas, que omitiam da equipe o uso de SPA e por conta da equipe das UAPS, por se sentir insegura quanto à assistência a essa população, preferindo o encaminhamento para o Centro de Atenção Psicossocial Álcool e Outras Drogas (CAPS-AD) e ambulatórios de pré-natal de alto risco. Em ambos os casos, foi criado um obstáculo para realizar as intervenções apropriadas e acompanhamento dos casos.

\section{Discussão}

A observação dos dados apresentados demonstrou que $o$ atendimento às gestantes em uso de SPA se deu na APS e nos serviços especializados, por meio do encaminhamento ao CAPS-AD e consultas intercaladas entre ambulatórios. Essa informação corrobora com estudos que objetivam a qualidade da assistência, a intersetorialidade e a integralidade como marco ético ${ }^{(5,13)}$.

A APS cumpre seu papel de articuladora da Rede de Atenção à Saúde (RAS) quando compartilha o cuidado com outros serviços de saúde especializados. A integralidade destaca-se como fio condutor e norteador do acompanhamento às gestantes classificadas como de alto risco. Em condições de uso de SPA, o cuidado demandará atenção profissional especializada às necessidades de saúde da gestante, que precisam ser identificadas no pré-natal. Assim, o cuidado integral garantirá a identificação de demandas em diferentes níveis de cuidado ${ }^{(9)}$.

A partir do momento que os serviços lançam mão da RAS para atender a gestante usuária de SPA com qualidade, as equipes de saúde encontram desafios a superar, como os destacados nos dados, com os frequentes encaminhamentos e perda de vínculo. A proposta do compartilhamento da assistência em diferentes níveis, aqui discutido entre a atenção primária e secundária, demanda que a comunicação entre ambas seja muito mais que impressos assistenciais de referência e contra referência. Neste contexto, o modelo da Estratégia Saúde da Família (ESF) tem a maior chance da captação precoce para o pré-natal e o acompanhamento compartilhado no alto risco(14).

A intercomunicação entre os serviços de ambulatório de pré-natal de risco habitual, de gestação de altorisco e o CAPS-AD funcionará como rede de apoio para evitar complicações na gestação em acompanhamento. Também proporcionará a construção de fluxos de atendimento às usuárias, beneficiando a todos, pois os cuidados de pré-natal devem ser redobrados e 
envolver diversos profissionais da área de saúde como nutricionistas, psicólogos, médicos e enfermeiros ${ }^{(15)}$.

A equipe da ESF nas UAPS onde a gestante iniciou o acompanhamento de pré-natal precisa manter o vínculo com esta mulher e sua família para estimular a adesão ao acompanhamento/tratamento, pois a APS não deve perder o papel crucial de coordenadora do cuidado(5,16). Esta equipe deve ser mantida informada a respeito da evolução da gravidez e tratamentos adotados com a gestante, por meio do contato entre as equipes, busca ativa e visita domiciliar ${ }^{(15)}$. Entretanto, os dados evidenciaram alguns desafios, como quando os enfermeiros referiram a perda de contato com as gestantes e desconhecimento sobre como proceder.

Além do ambulatório de alto risco, as gestantes atendidas nas UAPS também foram encaminhadas aos serviços especializados de saúde mental, os CAPS-AD, como afirmaram alguns enfermeiros. Estes serviços são responsáveis pela elaboração, com a gestante e família, de estratégias para o enfrentamento da abstinência das SPA ou medidas de redução de danos, na perspectiva da autonomia e corresponsabilização do cuidado(17-19).

Essa comunicação entre os serviços especializados em saúde mental e a UAPS é denominada apoio matricial, uma ferramenta de articulação em que equipes de referência do CAPS-AD podem subsidiar ações junto às equipes da APS, em um processo de construção compartilhada para o acompanhamento das gestantes e suas famílias ${ }^{(20)}$.

Quanto ao uso de SPA, segundo os enfermeiros, as mais frequentes entre as gestantes foram a maconha, tabaco, cocaína e crack; o álcool foi menos frequente. Estes dados reforçaram a importância da identificação precoce de fatores de risco, seu seguimento para a melhoria da qualidade de vida da mãe e do feto, bem como as prováveis complicações obstétricas e problemas cognitivos para a criança, caso essas informações sejam omitidas durante o pré-natal(21-22). As gestantes devem ser orientadas a não utilizar nenhum tipo de droga durante a gestação e amamentação, e fazer desse momento uma oportunidade de abandono da SPA(23-24).

Os dados também revelaram que as ações em torno do pré-natal e das atividades educativas desenvolvidas por enfermeiros junto às gestantes e seus familiares nas UAPS respeitam a diversidade de ações propostas pelo Ministério da Saúde, que criou o Programa de Assistência Integral à Saúde da Mulher (PAISM). Foram propostas ações educativas, com destaque do enfermeiro como principal responsável por estas atividades, já que esse é o profissional com maior proximidade das gestantes e possibilidade de repercussão positiva nelas(25-26).

O PAISM é voltado à promoção da saúde das gestantes no intuito de favorecer o ciclo gravídicopuerperal. Para sua boa condução, é imprescindível que a gestante, acompanhantes e familiares participem de grupos educativos e de apoio, pois constituem o foco principal do processo de aprendizagem. Além disso, estes grupos são momentos de ampliação do conhecimento das gestantes sobre seu corpo, condições de saúde, práticas preventivas, direitos e deveres. Assim, é possível evitar complicações maternas e fetais, de modo que o pré-natal, parto e puerpério transcorram de maneira mais tranquila e segura, e promovam a participação da gestante nas decisões acerca da sua saúde e processo reprodutivo(27).

Conforme os resultados encontrados, algumas gestantes possuíam dificuldades em admitir ou referir que utilizavam álcool ou outras drogas. Essa omissão pode estar impulsionada por medo e desconfiança(28-29) e dificulta o manejo profissional dos riscos reais durante o pré-natal, diminuindo as chances de aproximação e propostas de tratamento(27).

Diante disso, o momento da consulta de enfermagem de pré-natal é oportuno para discutir os malefícios do uso das SPA e as repercussões de cada doença sobre o organismo materno, fetal e do recém-nascido que podem ocorrer em decorrência desse consumo. O prénatal é o momento para esclarecer dúvidas e revisar e reforçar orientações relacionadas à gestação para a mulher e família. O enfermeiro, ao desenvolver ações educativas realizando grupos de forma dinâmica, envolve os participantes em uma discussão que Ihes propicia a exposição de suas dúvidas em ambiente de acolhimento e prevenção(30).

Com base nas dificuldades apresentadas pelos enfermeiros, o desenvolvimento de habilidades de cuidado terapêutico em torno dos riscos do uso de substâncias psicoativas durante a gestação e com o objetivo de promover a saúde e prevenir agravos constitui um desafio para os profissionais de saúde. O trabalho com essas gestantes exige um processo de crescimento e aquisição de novas competências, como conhecimentos, habilidades e atitudes para os dois protagonistas do processo: enfermeiro e gestantes ${ }^{(27,30)}$.

Para conhecer o comportamento em relação à saúde e a doença, os profissionais devem educar para a saúde e estar abertos para escolher e escutar ${ }^{(4)}$. A problemática da adesão ao tratamento e os fatores relacionados a gestantes em uso de SPA são complexos, pois envolvem sexo, idade, escolaridade, nível socioeconômico, cronicidade e a sintomatologia da doença, crenças, hábitos culturais e de vida, custos, efeitos indesejáveis e esquemas complexos de tratamento, relacionamento com a equipe de saúde e um esforço muito grande dos profissionais no sentido de que as gestantes sejam assíduas em qualquer forma de atendimento(30).

Esses apontamentos evidenciaram a necessidade de capacitação dos enfermeiros atuantes na atenção à saúde da mulher, dada a ampla gama de ações que poderão estar 
alinhadas com uma perspectiva dialógica e orientadas para o reconhecimento do outro e da sua singularidade(13).

A educação para a saúde da mulher gestante é fundamental para prevenir e controlar complicações durante a gravidez, o parto e o puerpério. Uma RAS voltada para ações e serviços articulados em níveis de complexidade crescentes é de suma importância. A rede deve atuar de acordo com suas especificidades e enfatizar serviços de base comunitária adequados às necessidades do usuário e seus familiares, já que atitudes isoladas e fragmentadas não atendem as demandas. Nessa esfera, as contribuições da enfermagem são educar a gestante e fortalecer a sua consciência sobre o valor da saúde, bem como promover o cuidado, implementar intervenções, avaliar resultados e considerar necessidades e anseios ${ }^{(31)}$.

A realização desta atividade pelo profissional enfermeiro está amparada na lei do exercício profissional (lei $n^{\circ} 7498 / 86$ ) e respaldada pela regulamentação municipal. Estas preconizam a realização da consulta de enfermagem no pré-natal, com vistas a garantir assistência de enfermagem à gestante, parturiente e puérpera, bem como a educação em saúde com o propósito de promover a melhoria da saúde da população. Neste sentido, os enfermeiros poderão lançar mão de uma assistência integral às gestantes e abordar ações educativas em saúde, visando a melhoria da saúde desta clientela( ${ }^{(30)}$.

\section{Limitações do estudo}

Um fator limitante do estudo foi a população ter sido composta apenas por enfermeiros das seis UAPS pertencentes ao Projeto Integrador Ensino e Pesquisa da Faculdade Suprema-Sociedade Universitária para o Ensino Médico. Tal fato impossibilitou conhecer todas as estratégias e condutas dos enfermeiros com gestantes em uso de SPA de todas as UAPS. Não dar voz às gestantes e aos seus familiares também incorreu em limitação.

\section{Conclusão}

Neste estudo, foi observado que a temática SPA/ gestação ainda é um obstáculo e os profissionais devem desenvolver uma visão crítica ante a problemática no seu cotidiano. Ao mesmo tempo, eles não devem ser moralistas em relação aos problemas e desafios a serem enfrentados com essa demanda, que vem aumentando gradativamente no país.

O enfermeiro é o profissional que permanece mais tempo em contato com essa clientela e em suas mãos estão a oportunidade e o desafio de minimizar e diagnosticar o uso de SPA.

Por meio desse estudo, foi fácil perceber o mínimo interesse e participação das gestantes. Sua baixa circulação nos programas específicos revela a problemática dessa questão. Os relatos da baixa adesão de gestantes usuárias de SPA em acompanhamento também revelam graves problemas de saúde pública, que na maioria das vezes são banalizados.

A criação de vínculo com as gestantes é de extrema importância, e a APS deve se colocar próximo e disponível para tal, de forma que as mesmas se sintam acolhidas e queiram fazer parte de grupos educativos para trocas de experiências e interação, efetivando vínculo com a UAPS e favorecendo a adesão ao pré-natal.

Este estudo alcançou o objetivo proposto, mas enfatiza-se a importância do trabalho em equipe multidisciplinar, pois a percepção de cada membro é agregadora para o manejo, acompanhamento e direção para cada situação que surgir. Os resultados deste estudo podem auxiliar futuras pesquisas quanto ao fortalecimento das ações desenvolvidas por enfermeiros e outros profissionais na APS.

\section{Referências}

1. O'Keeffe LM, Kearney PM, McCarthy FP, Khashan AS, Greene RA, North RA et al. Prevalence and predictors of alcohol use during pregnancy: findings from international multicentre cohort studies. BMJ Open. [Internet]. 2015; 5(7). [cited Dez 4 2019]. doi: 10.1136/ bmjopen-2014-006323

2. Kroeff LR, Mengue SS, Schimidt MI, Duncan BB, Favaretto ALF. Correlates of smoking in pregnant women in six Brazilian cities. Rev Saude Publica. [Internet]. 2004; 38(2):261-267. [cited Nov 2 2019]. Available from: https://doi.org/10.1590/S0034-89102004000200016

3. Guimarães VA, Fernandes KS, Lucchese R, Vera I, Martins BCT, Amorim TA et al. Prevalence and factors associated with alcohol use during pregnancy in a maternity hospital in Goiás, Central Brazil. Ciência \& saúde Coletiva, 23 (10):3413-3420, 2018. [cited Mar 27 2020]. https://doi.org/10.1590/1413-812320182310.24582016 4. Ribeiro MCL, Giusti BB, Ciosak SI, Silva IM. Healthcare of women crack users during pregnancy: bibliographic review. SMAD, Rev. Eletrônica Saúde Mental Álcool Drog. 2018 Jul.-Set.;14(3):185-193. [cited Mar 27 2020]. http://pepsic.bvsalud.org/pdf/smad/v14n3/09.pdf

5. Brasil. Ministério da Saúde. Secretaria de Atenção à Saúde. Departamento de Atenção Básica. Atenção ao Pré-Natal de Baixo Risco. Brasília: Editora do Ministério da Saúde, 2012. [cited Dez 15 2019]. Available from: http://bvsms.saude.gov.br/bvs/publicacoes/cadernos_ atencao_basica_32_prenatal.pdf

6. Lima LPM, Santos AAP, Póvoas FTX, Silva FCL. Nurse's role during prenatal consultation of pregnant drug users. Rev Espaço para a Saúde. 2015 jul-set; 16(3):39-46. [cited Dez 5 2019]. Available from: http://espacoparasaude.fpp. edu.br/index.php/espacosaude/article/viewFile/394/382 
7. Brasil. Ministério da Saúde. Secretaria de Atenção à Saúde. Departamento de Ações Programáticas Estratégicas. Gestação de alto risco: manual técnico. 5ed. Brasília: Editora do Ministério da Saúde; 2012. Available from: http://bvsms.saude.gov.br/bvs/publicacoes/ manual_tecnico_gestacao_alto_risco.pdf

8. Monte MG, Sérvio VRFT, Santos PAM, Maia JA. Effects of illicit drug use during pregnancy. Dêciência em Foco 2017 p. 95-105. [cited 28 Mar 2020]. Available from: http://revistas.uninorteac.com.br/index.php/ DeCienciaemFoco0/article/view/77/33

9. Cabrita, BAC, Abrahão AL, Rosa AP, Freitas FCF. The search for care by high risk pregnant in relation to integrality in health. Cienc Cuid Saude [Internet] 14.2 (2015): 1139-78. [cited Dez 4 2019]. http://periodicos. uem.br/ojs/index.php/CiencCuidSaude/article/ view/24250/pdf_347

10. Minayo MCS. O desafio do conhecimento: pesquisa qualitativa em saúde. Hucitec/Abrasco. São Paulo, ed. 14, p. 407, 2014. 416 p.

11. Triviños NA. Introdução à pesquisa em ciências sociais: a pesquisa qualitativa em educação. São Paulo (SP): Atlas; 2013. 176 p.

12. Bardin L. Análise de conteúdo. São Paulo: Edições 70, 2016. $141 \mathrm{p}$

13. Marcolino TQ, Joaquim RHVT, Giovanetti G, Kishi RGB. Marchi M, Fejes MAN et al. Pregnancy and drugs: what healthcare do women want. Cad. Saúde Colet., 2018, Rio de Janeiro, 26 (3): 255-260. [cited 28 Mar 2020]. doi 10.1590/1414-462X201800030374

14. Sanine PR, Venancio SI, Silva FLG, Aratani N, Mota MLG et al. Prenatal care in high-risk pregnancies and associated factors in the city of São Paulo, Brazil. Cad. Saúde Pública 2019; 35(10):e00103118. [cited 27 Marc 2020]. https://doi.org/10.1590/0102-311x00103118 15. Guimarães WSG, Parente RCP, Guimarães TLF, Garnelo L. Access to prenatal care and quality of care in the Family Health Strategy: infrastructure, care, and management. Cad. Saúde Pública 2018; 34(5):e00110417. [cited 28 Mar 2020]. doi: 10.1590/0102-311X00110417

16. Starlfield B. Atenção primária: equilíbrio entre necessidades de saúde, serviços e tecnologia. Brasília: UNESCO, Ministério da Saúde; 2002. Available from: https://repositorio.observatoriodocuidado.org/handle/ handle/2326

17. Machado LV, Boarini ML. Drug policies in Brazil: the harm reduction strategy. Psicol. Cienc. Prof. 2013;33(3):580-95. [cited 27 Mar 2020]. Available from: https://doi.org/10.1590/S1414-98932013000300006

18. Ronzani TM. Ações Integradas sobre drogas: prevenção, abordagens e políticas públicas. Juiz de Fora: EDUFJF; 2013. 448 p.

19. Galassi $A D$, Santos V. The necessary and urgent changing in the approach toward people in suffering by drug use. Cad Ter Ocup UFSCar. 2014;22(1):1-4. [cited 28 Mar 2020]. Available from: http://dx.doi.org/ $10.4322 /$ cto. 2014.024

20. Brasil. Ministério da Saúde. Centro de Estudo e Pesquisa em Saúde Coletiva. Chiaverini DH. Guia prático de matriciamento em saúde mental. I. Brasília DF: Ministério da Saúde; 2011. Available from: http://bvsms.saude. gov.br/bvs/publicacoes/guia_pratico_matriciamento_ saudemental.pdf

21. Antunes MB, Demitto MO, Padovani C, Elias KCME, Miranda ACM, Pelloso SM. Perinatal outcomes in pregnant drug users attended at a specialized center. SMAD Revista Electronica Salud Mental, Alcohol y Drogas 2018; 14.4. [cited 27 Mar 2020]. Available from: http:// pepsic.bvsalud.org/scielo.php?script=sci_arttext\&pi $\mathrm{d}=\mathrm{S} 1806-69762018000400004$

22. Maia JA, Pereira LA, Menezes FA. Consequences of drug use during pregnancy. Revista Enfermagem Contemporânea. 2015 Jul./Dez.;4(2):121-128. [cited 27 Mar 2020]. doi: http://dx.doi.org/10.17267/23173378rec.v4i2.664

23. Rocha ENT, Rocha RR. Drugs in pregnancy and consequences in newborns. Journal of Specialist. v.2, n.2,p.1-29, Abr -Jun, 2018.[cited 28 Mar 2020]. Available from: http://journalofspecialist.com/jos/index. php/jos/article/view/81/39

24. Justi DLT, Júnior JBL, Comandule AQ, Morton ES. Marijuana and pregnancy: cannabis hyperemesis syndrome - Case report. J Bras Psiquiatr. 2018;67(1):59-62. [cited 28 Mar 2020]. doi: https://doi.org/10.1590/ 0047-2085000000185

25. Rodrigues DP, Guerreiro EM, Ferreira MA, Queiroz ABA, Barbosa DFC, Fialho AVM. Social representations of women in pregnancy, postpartum, and educational actions. Online braz j nurs [Internet]. 2013 [cited 2020 Mar 28];12(4):618-27. Available from: http://www.objnursing. uff.br/index.php/nursing/article/view/4287/html_24

26. Quental LLC, Nascimento LCCC, Leal LC, Davim RMB, Cunha ICBC. Educational practices with pregnant women at a primary health care. Rev enferm UFPE on line., Recife, 11(Supl. 12):5370-81, dez., 2017. [cited 28 Mar 2020]. Available from https://doi.org/10.5205/19818963-v11i12a23138p5370-5369-2017

27. Almeida FS, Oliveira RAF, Coelho EOE. The importance of prenatal care by professional nurse. REMAS-Revista Educação, Meio Ambiente e Saúde, 2016, 6.2: 155-169. [cited Dez 4 2019]. Available from: http://www. faculdadedofuturo.edu.br/revista1/index.php/remas/ article/view/37

28. Schempf AH, Strobino DM. Drug use and limited prenatal care: an examination of responsible barriers. Am J Obstet Gynecol. 2009; 200(4):412.e1-10. [cited 27 Mar 2020]. doi: 10.1016/j.ajog.2008.10.055

29. Portela GLC, Barros LM, Frota NM, Landim APP, Caetano JÁ, Farias FLRF. Perception of pregnant on consumption of illicit drugs in pregnancy. SMAD, 
Rev Eletrônica Saúde Mental Álcool Drog. 2013 [cited 2020 Mar 28]; 9(2):58-63. Available from: http://pepsic.bvsalud. org/scielo.php?script=sci_arttext\&pid=S1806-69762 013000200002\&lng=pt\&nrm=iso\&tlng=pt

30. Amorim TV, Souza IEO, Moura MAV, Queiroz ABA, Salimena AMO. Nursing care perspectives in highrisk pregnancy: integrative review. Enfermería Global, 2017, 16.2: 500-543. [cited Dez 4 2019]. Available from: http://scielo.isciii.es/pdf/eg/v16n46/pt_16956141-eg-16-46-00500.pdf

31. Souza EVA, Bassler TC, Taveira AG. Health education in the empowerment of the pregnant woman. Rev enferm UFPE on line., Recife, 13(5):1527-31, maio., 2019. [cited 28 Mar 2020]. Available from https://periodicos.ufpe.br/ revistas/revistaenfermagem/issue/view/2756

\section{Contribuição dos autores}

Concepção e desenho da pesquisa: Angela Aparecida Peters, Giselle de Paula Assis, Hugo Ramalho Cruzeiro, Otávia Gonçalves Paulino Bertolini, Adriana Dias Silva, Maria Angélica de Almeida Peres. Obtenção de dados: Angela Aparecida Peters, Otávia Gonçalves Paulino, Hugo Ramalho Coelho, Maria Angélica de Almeida Peres. Análise e interpretação dos dados: Angela Aparecida Peters, Hugo Ramalho Coelho, Otávia Gonçalves Paulino, Giselle de Paula Assis, Adriana Dias Silva, Maria Angélica de Almeida Peres. Redação do manuscrito: Angela Aparecida Peters, Giselle de Paula Assis, Adriana Dias Silva, Maria Angélica de Almeida Peres, Hugo Ramalho Coelho, Otávia Gonçalves Paulino. Revisão crítica do manuscrito quanto ao conteúdo intelectual importante: Angela Aparecida Peters, Adriana Dias Silva, Maria Angélica de Almeida Peres, Giselle de Paula Assis.

Todos os autores aprovaram a versão final do texto.

Conflito de interesse: os autores declararam que não há conflito de interesse. 